\title{
Evaluation of Two Lactic Acid Bacteria Starter Cultures for the Fermentation of Natural Black Table Olives (Olea europaea L cv Kalamon)
}

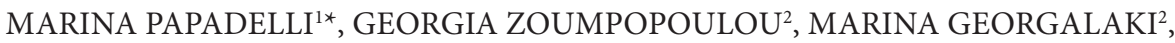 \\ RANIA ANASTASIOU², EUGENIA MANOLOPOULOU², IOANNA LYTRA ${ }^{1}$, \\ KONSTANTINOS PAPADIMITRIOU² and EFFIE TSAKALIDOU ${ }^{2}$
}

\author{
${ }^{1}$ Technological Educational Institute of Peloponnese, Department of Food Technology, Kalamata, Greece \\ ${ }^{2}$ Agricultural University of Athens, Department of Food Science and Human Nutrition, Athens, Greece
}

Submitted 2 January 2015, revised 22 February 2015, accepted 5 March 2015

\begin{abstract}
The production of Greek-style natural black table olives remains an empirical process relying on spontaneous fermentation despite its economic significance. For this reason producers often resort to increased $\mathrm{NaCl}$ concentration of the brine to secure quality of the product. In this study we employ two lactic acid bacteria Leuconostoc mesenteroides subsp. mesenteroides Lm139 and Lactobacillus pentosus DSM 16366 as starters in separate laboratory low salinity fermentations of "Kalamon" cultivar olives, processed according to the Greek-style method. L. mesenteroides subsp. mesenteroides Lm139 was previously isolated from Kalamon olives laboratory spontaneous fermentations, while L.pentosus DSM 16366 was isolated from fermenting green olives prepared according to the Spanish-style method. Spontaneous olives fermentation was also performed as a control. Microbiological and physicochemical analyses of the brines revealed that the use of the starters had a significant effect on the olives fermentation, leading to a faster acidification due to the more efficient consumption of soluble sugars in the brines. The final $\mathrm{pH}$ value reached by each starter culture used indicates a successful lactic fermentation. The production of lactic acid by the starters and the concomitant drop of the $\mathrm{pH}$ value proved to inhibit enterobacteria in a shorter period of time compared to the spontaneous fermentation. Concluding, the use of either of the two lactic acid bacteria as starters in Greek-style Kalamon olives fermentation could lead to a more controllable fermentation at lower salinities. The resulting product could be of higher quality with extended shelf-life while being at the same time safer for the consumer.
\end{abstract}

Ke y w o r d s: fermentation, Greek-style olives, Kalamon olives, lactic acid bacteria

\section{Introduction}

For thousands of years, table olives have been a basic component of the Mediterranean diet. They have high nutritional value, being rich in energy, minerals and vitamins, and they possess antioxidant properties. The estimated Greek production of table olives for the period 2011/12 was about 130,000 tons, the $2^{\text {nd }}$ (after Spain) in the EU which corresponds to the $17.5 \%$ of the total EU production (International Olive Council, 2012a; 2012b).

"Kalamata type table olives" is a very well known type of Greek-style natural black olives, mainly due to the excellent characteristics of the raw material used, which is the Greek olive cultivar "Kalamon". The processing method for their production remains artisanal and empirical despite its economic importance. According to the common practice applied by the producers, the "Kalamon" olives are placed in brine of high salt concentration (usually $6-10 \% \mathrm{w} / \mathrm{v} \mathrm{NaCl}$, but very often even higher, $10-14 \% \mathrm{w} / \mathrm{v} \mathrm{NaCl}$ ) and they are spontaneously fermented in brine by a mixed population of indigenous microorganisms, mainly yeasts and lactic acid bacteria (LAB). In the final step of the preparation, fermented black olives are size graded before being packed also in brine solution (Balatsouras, 1967; 1990; Garrido-Fernández et al., 1997).

The use of high salinity brine ensures the inhibition of spoilage microbiota and the growth of pathogens in the brines, but it also leads to a slow extraction of soluble components from the olives to the brine. This means a slower removal of bitterness but also a longer fermentation period of olives, lasting even 10 or more months. Furthermore, in such a high salinity ( $>8 \% \mathrm{NaCl}$ in equilibrium), yeasts dominate over LAB which are prevented to grow and thus cannot assist in the fermentation process (Arroyo-López et al., 2008). When yeasts are dominant the product has a milder

* Corresponding author: M. Papadelli, Technological Educational Institute of Peloponnese, Department of Food Technology, Antikalamos, 24-100 Kalamata, Greece; e-mail: m.papad@teikal.gr 
taste and a shorter shelf life (Garrido-Fernández et al., 1997; Panagou et al., 2008). More precisely, an incomplete yeast fermentation, leading to the remaining of residual sugars in the brine even after a long fermentation period, may result in future undesirable secondary fermentations during the storage of the olives. Furthermore, yeasts may cause gas pocket formation, softening of the olive tissue, package bulging, clouding of the brines and production of off flavors and odours (Tofalo et al., 2012). Finally, the olives produced by fermentation in high salinity brines are usually shrunk with high salt content and with a remaining bitterness.

In order to reduce the above mentioned negative consequences of the high salt content and yeast domination, a more active role of LAB in olives fermentation is required. For this purpose, during this study, laboratory fermentations using specific LAB strains as starter cultures were performed in the presence of low salt concentration $(5 \% \mathrm{w} / \mathrm{v} \mathrm{NaCl})$.

\section{Experimental}

\section{Materials and Methods}

Olive fermentation. The experimental olive fermentations were carried out with olives Olea europaea L. cv. "Kalamon", kindly provided by a table olive producer from Petalidi village in Messinia area, Peloponnese. The olive fruits were collected during November 2009 , transported within $24 \mathrm{~h}$ to the laboratory, and washed with tap water in order to remove the dust and the impurities. The olives were once cut and left in tap water at room temperature for 24 hours. After that period, nine lidded plastic fermentation vessels were set up, each of them containing $1,200 \mathrm{~g}$ of olives and $800 \mathrm{ml}$ of brine $(5 \% \mathrm{w} / \mathrm{v} \mathrm{NaCl})$. All fermentation vessels remained at $22^{\circ} \mathrm{C}$ for $48 \mathrm{~h}$ prior the inoculation.

Three sets of fermentations (B1, B2, B3) were performed and each set was repeated three times. In fermentation B1, no inoculation was applied and a spontaneous fermentation was leaded by the indigenous microbiota. In fermentation B2, the vessels were inoculated with the Lactobacillus pentosus DSM 16366 strain (Delgado et al., 2005), which was purchased from the DSMZ collection (Germany). In fermentation B3 the vessels were inoculated with a wild strain of L. mesenteroides subsp. mesenteroides (Lm139) isolated from a spontaneous Kalamon olives fermentation. In both fermentations B2 and B3, the inocula were taken from overnight cultures in MRS broth supplied with $5 \% \mathrm{w} / \mathrm{v} \mathrm{NaCl}$, for a better adaptation of the bacteria to the salinity in the cover brine. The cells were collected by centrifugation at $10000 \times \mathrm{g}$ at $4^{\circ} \mathrm{C}$, resuspended in $0.9 \% \mathrm{w} / \mathrm{v} \mathrm{NaCl}$ and inoculated to $10^{6} \mathrm{cfu} \times \mathrm{ml}^{-1}$ of cover brine solution.
All fermentation vessels were incubated at $22^{\circ} \mathrm{C}$ for $70 \mathrm{~d}$. During that period, the salt concentration of the brine was periodically monitored and adjusted to the initial level of $5 \%$. Brine samples were collected at day 0 (the sample was taken about $30 \mathrm{~min}$ after the inoculation), $3,5,7,10,13,17,21,30,40$ and $70 \mathrm{~d}$ of fermentation, for microbiological and physicochemical analyses.

Microbiological analysis. Samples (brine or olives) were serially diluted with a factor of 10 in saline solution $(0.9 \% \mathrm{w} / \mathrm{v})$ and plated in triplicate on selective agar media (Biokar Diagnostics Beauvais, France) in order to enumerate the following microbial groups: (i) LAB on double layer of de Man-Rogosa-Sharp (MRS) $\mathrm{pH} 5.6$, modified by the addition of $500 \mu \mathrm{g} \mathrm{ml}^{-1}$ cyclohexamide, incubated at $30^{\circ} \mathrm{C}$ for $48 \mathrm{~h}$, (ii) yeasts and moulds on Yeast Extract Glucose Chloramphenicol (YGC) incubated at $25^{\circ} \mathrm{C}$ for $72 \mathrm{~h}$ and, (iii) enterobacteria on double layer of Violet Red Blue Lactose (VRBL) incubated at $30^{\circ} \mathrm{C}$ for $24 \mathrm{~h}$. The same microbial groups were enumerated in fresh (raw) olive fruits before their immersion in tap water, by dilution of $10 \mathrm{~g}$ of olives in $90 \mathrm{ml}$ of saline solution $(0.9 \% \mathrm{w} / \mathrm{v})$, decimal dilutions in the same saline solution and plating on the above mentioned selective agar media.

Physicochemical analyses. The $\mathrm{pH}$ of the brine samples was measured using a Metrohm model $827 \mathrm{pH}$ meter (Herisau, Switzerland). Titratable acidity of the brines was determined by titration with $0.1 \mathrm{~N} \mathrm{NaOH}$ up to $\mathrm{pH} 8.2$ and the result was expressed as $\%$ of lactic acid $(\mathrm{w} / \mathrm{v})$.

Total phenolics in the brine as well as in the fruit flesh were quantified by the Folin-Ciocalteau assay (Singleton and Rossi, 1965) with the adaptations for the olive fruit applied by Marsilio et al. (2005).

Organic acids (lactic, succinic, malic, acetic, citric and tartaric) as well as sugars (glucose, sucrose and fructose) and sugar alcohols (mannitol) in the brine were determined by HPLC (LC 1150 HPLC Pump, GBC Scientific Equipment, Dandenong, Victoria, Australia). One $\mathrm{ml}$ of the brine sample was overnight precipitated with $\mathrm{HClO}_{4}$ (final concentration $6.4 \%$ ) at $4^{\circ} \mathrm{C}$, centrifuged $\left(12000 \times \mathrm{g}\right.$ for $1 \mathrm{~h}$ at $\left.4{ }^{\circ} \mathrm{C}\right)$ and the supernatant was filtered $(0.2 \mu \mathrm{m}$ filters $)$. A $20-\mu \mathrm{l}$ sample of the filtrate was injected into an Aminex HPX-87H column (300 by $7.8 \mathrm{~mm}$; Bio-Rad, Hercules, CA, USA) connected to a refractive index detector (model LC 1240; GBC Scientific Equipment). Elution was performed at $65^{\circ} \mathrm{C}$ with $4.5 \mathrm{mM} \mathrm{H}_{2} \mathrm{SO}_{4}$ at a flow rate of $0.5 \mathrm{ml} \mathrm{min}^{-1}$. Data acquisition and processing were performed by the Win Chrom $^{\mathrm{TM}}$ Chromatography Data Acquisition Software v. 1.32 (GBC Scientific Equipment). The quantification analysis of the above organic acids and sugars was performed from standard curves, which were prepared from different concentrations of the respective reference substances analyzed under the same conditions. 
Statistical analysis. Differences among means of measurements were assessed with the analysis of variance (ANOVA). Post-hoc analysis was performed with the Tukey's test for $\mathrm{p}<0.05$.

\section{Results and Discussion}

The aim of this study was to evaluate the potential of the wild strain L. mesenteroides subsp. mesenteroides Lm139, isolated from the brine of Kalamon olives laboratory spontaneous fermentation, as a starter in Kalamon table olives fermentation, in low salinity brines. The choice of this autochthonous LAB strain was based on its tolerance to $1 \% \mathrm{w} / \mathrm{v}$ oleuropein - the bitter glucoside found in olives - and to high salt concentration $(10 \% \mathrm{w} / \mathrm{v} \mathrm{NaCl})$, as well as to its ability to hydrolyse the oleuropein, as it produces the $\beta$-glucosidase enzyme (data not shown). Lactobacillus pentosus DSM 16366, isolated from fermenting green olives of "Azeiteria" cultivar prepared according to Spanish style (Delgado et al., 2005), was also examined as an alternative starter culture. This strain is considered to be a strain with technological interest which could be used as a starter for table olives, since it produces the bacteriocin plantaricin $S$ in environmental conditions resembling green olives fermentation (Delgado et al., 2005). It has been additionally successfully used in experimental green olives Spanish-type fermentation by Peres et al. (2008).

Evolution of microbial populations during fermentation. Microbiological analysis of fresh/raw Kalamon olives used in the experimental fermentations, after washing and before their placement in tap water, resulted in the following microbial populations: $\mathrm{LAB}<10 \mathrm{cfu} \mathrm{g}^{-1}$, yeasts and moulds $1.3 \times 10^{3} \mathrm{cfu} \mathrm{g}^{-1}$ and enterobacteria $<10 \mathrm{cfu} \mathrm{g}^{-1}$.

The population dynamics of the three microbial groups (LAB, yeasts/moulds, enterobacteria) examined in the brines of the three olive fermentations is presented in Fig. 1. All three microbial groups exhibited
Fig. 1. Population dynamics of lactic acid bacteria (A), yeasts/moulds (B) and enterobacteria (C) in brines, during the 70 days of $\mathrm{cv}$. Kalamon olives fermentation.

$(\triangleleft)$ : spontaneous fermentation B1, (®): fermentation B2, inoculated with L.pentosus DSM 16366, and ( $\mathbf{\Delta})$ : fermentation B3, inoculated with L.mesenteroides subsp. mesenteroides Lm139. Data reported are means from three repetitions \pm standard deviation.
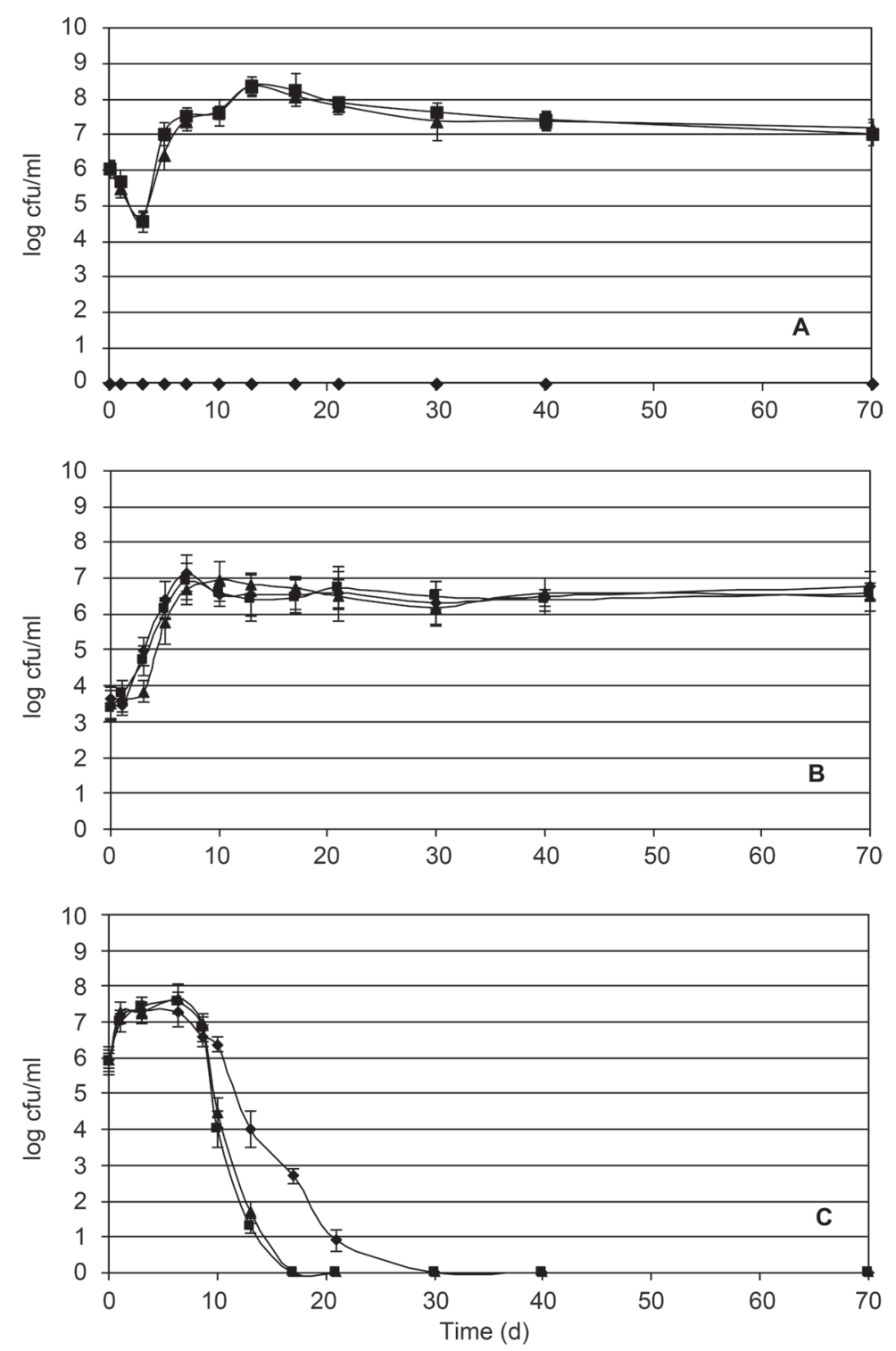


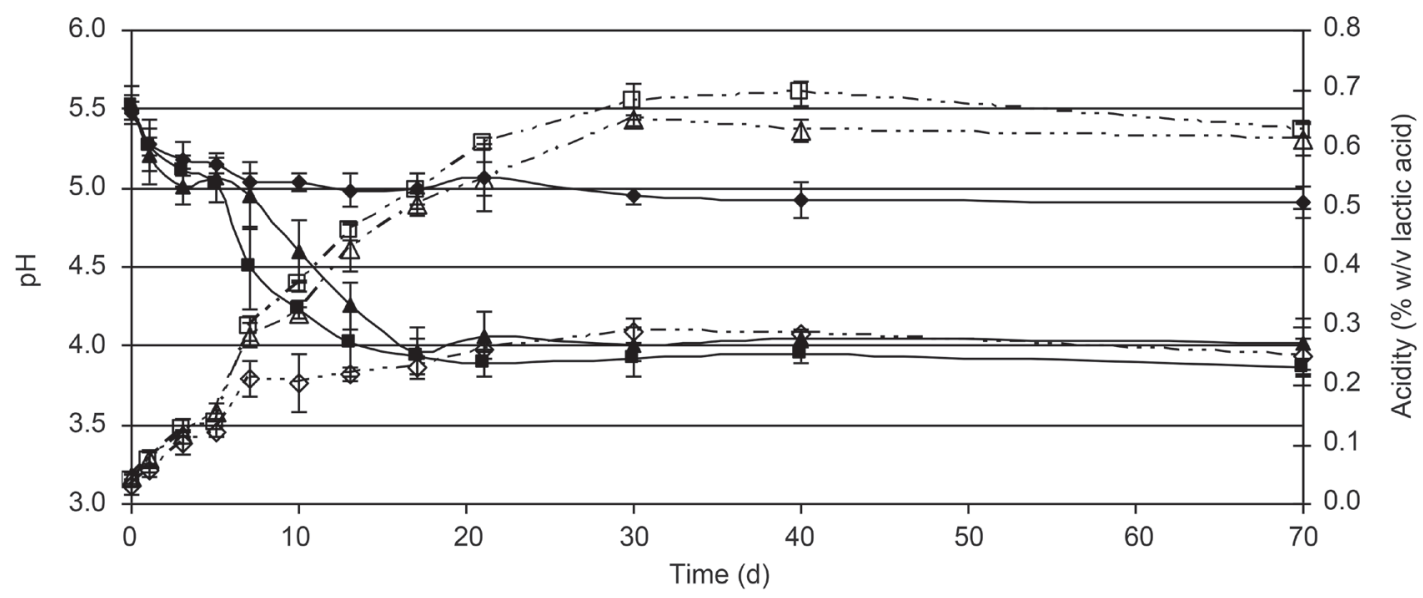

Fig. 2. Changes in $\mathrm{pH}$ (solid symbols) and titratable acidity (empty symbols, dashed lines) of brines, during the 70 days of cv. Kalamon olives fermentations.

): spontaneous fermentation B1, ( $\mathbf{\square})$ : fermentation B2, inoculated with L.pentosus DSM 16366, and ( $\mathbf{\Delta}$ ): fermentation B3, inoculated with L. mesenteroides subsp. mesenteroides Lm139. Data reported are means from three repetitions \pm standard deviations.

growth in the inoculated fermentation brines (B2 and B3 inoculated with L.pentosus DSM 16366 and L. mesenteroides subsp. mesenteroides Lm139, respectively). In contrast, in the brines of the non-inoculated spontaneous fermentation B1, LAB were not detected at all throughout the fermentation period of $70 \mathrm{~d}$ (Fig. 1a).The growth profile of LAB in brines B2 and B3 revealed that after a 3-day initial phase of adaptation, LAB managed to recover, and their population increased by 2 logs on day 13, compared to the initial inoculum in the brines (Tukey's test, $\mathrm{p}<0.05$ ). Afterwards, the population plateaued for several days followed by a slow decrease that reached an approximately $1 \log$ reduction at day 70 in comparison to the population observed at day 13. No significant differences were observed among the growth patterns of the yeast populations in fermentations B1, B2 and B3 (Fig. 1b). In the spontaneous fermentation $B 1$, growth of yeasts and moulds was not stimulated by the absence of competition from the LAB population and enterobacteria were eliminated after $30 \mathrm{~d}$, while in fermentations B2 and B3 the use of the starter cultures influenced the population of enterobacteria that disappeared much earlier, on day 13 and 17, respectively (Fig. 1c).

Physicochemical changes during fermentation. Figure 2 presents changes in $\mathrm{pH}$ and titratable acidity of brines in the three olive fermentations. In the spontaneous fermentation $\mathrm{B} 1$, the $\mathrm{pH}$ value of the brine dropped only to 4.9 (from the initial value of 5.5) at the end of the experiment (day 70), when the acidity reached only the value of $0.25 \%(w / v)$ lactic acid. On the contrary, in fermentations $\mathrm{B} 2$ and $\mathrm{B} 3$, the $\mathrm{pH}$ dropped within the first 17 days to the values of 3.9 and 4.0 respectively and remained almost constant until the day 70 . The corresponding values of the brines' acidity at the end of the experiment were $0.63 \%$ for B2 and $0.62 \%$ for $\mathrm{B} 3$ (day 70 ). Our findings suggest that the use of LAB starter cultures had a significant effect on the acidification of the brines. The amount of acid produced and the final $\mathrm{pH}$ value in fermentations $\mathrm{B} 1$ may not contribute enough to the preservation of the product. The higher acidity and the lower $\mathrm{pH}$ values of fermentations B2 and B3 are sufficient to inhibit the growth of spoilage microorganisms and thus they may provide an extended shelf life to the product. This could also be supported by the faster disappearance of enterobacteria in the latter fermentations as discussed above.

In the three fermentations, the phenolic content of the olives declines because of the diffusion of the compounds to the brine, and as a consequence the brines' phenolic concentration increases (data not shown). The phenolic content of the fresh olives used for all fermentations corresponded to about $20 \mathrm{mg}$ gallic acid $\times$ g olive $^{-1}$.

The concentration levels of organic acids in the brine during the fermentation process are presented in Fig. 3. The major organic acid detected during fermentations B2 and B3 was lactic acid (ranging from $99 \mathrm{mM}$ in $\mathrm{B} 3$ to $118 \mathrm{mM}$ in $\mathrm{B} 2$, after $70 \mathrm{~d}$ of fermentation), produced probably by the LAB growing in the brine. In the spontaneous fermentation B1, lactic acid was detected in traces throughout the 70 days. This result is in accordance with the absence of LAB observed in this fermentation (Fig. 1a).

The acidification of the inoculated brines was not only due to the production of lactic acid, but also due to the production of acetic acid. In fermentations B2 and B3, acetic acid was detected in lower concentrations compared to lactic acid, ranging from $18 \mathrm{mM}$ in $\mathrm{B} 3$ to $22 \mathrm{mM}$ in B2 after 70 days of fermentation. The presence of acetic acid in the brines of both $\mathrm{B} 2$ and $\mathrm{B} 3$ could be attributed to a shift from homo- to hetero- 

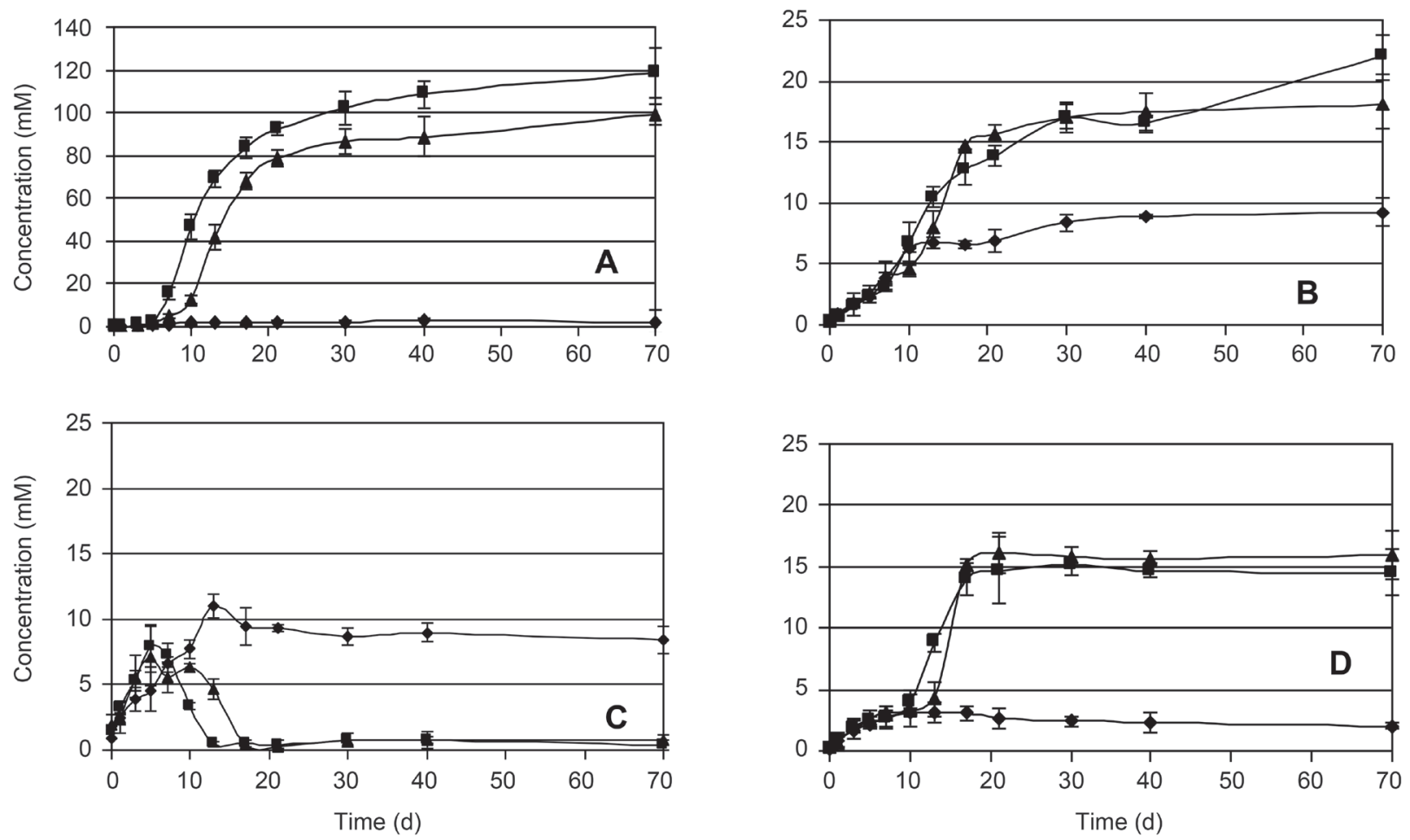

Fig. 3. Changes in the concentration (mM) of lactic acid (A), acetic acid (B), tartaric acid (C) and succinic acid (D) in brines, during the 70 days of cv. Kalamon olives fermentation.

): spontaneous fermentation B1, ( $\mathbf{\square})$ : fermentation B2, inoculated with L.pentosus DSM 16366, and ( $\mathbf{\Delta}$ ): fermentation B3, inoculated with L. mesenteroides subsp. mesenteroides Lm139. Data reported are means from three repetitions \pm standard deviations.

fermentative metabolism of L.pentosus and L. mesenteroides subsp. mesenteroides. In the non-inoculated brine $\mathrm{B} 1$, the presence of acetic acid remained lower $(9 \mathrm{mM}$ at day 70) compared to fermentations B2 and B3. Succinic acid was not detected in brine $\mathrm{B} 1$, while the evolution of its concentration in fermentations B2 and B3 was found to be similar to that of the acetic acid. Low concentration of tartaric acid was also detected in both inoculated samples during the first 10 days of fermentation; the concentration observed reached $7.9 \mathrm{mM}$ and $7.3 \mathrm{mM}$ at day 5 for fermentations B2 and B3, respectively. It was further decreased to almost zero on day 13 for both samples. On the contrary, in spontaneous fermentation B1, tartaric acid was constantly detected in the brine till the end of the fermentation $(8.4 \mathrm{mM}$ on day 70 ). The tartaric acid is referred as an acid found in olives and the observed increase of its concentration in the brines during the first days of fermentation is attributed to its extraction from the olives (Vlachov, 1976). It could be assumed that in fermentations B2 and B3 the microorganisms may have metabolised the acid.

Finally, HPLC analysis revealed either absence or presence in traces for malic and citric acid, in all three samples, during the entire process of fermentation (data not shown). This finding is in agreement with results reported by other authors (Tassou et al., 2002; Panagou et al., 2008; Tofalo et al., 2012), who detected malic acid at low amounts at the beginning of the process that decreased at the end of the fermentation period. According to the literature, the gradual decrease in the concentration of these two organic acids in brines observed during the fermentation of green olives is attributed to the microbial degradation of malic acid to lactic acid and $\mathrm{CO}_{2}$, as well as to the citric acid metabolism to acetic and succinic acid (Montano et al., 1993). However, in the current study, the absence of both acids from the brines even at day 0 , i.e. after two days of incubation of olives in the brine, means that these two acids were not extracted from the olives at all.

Soluble sugars extracted from olive fruits into the brine are the substrates for microbial fermentation, leading to the production of acids responsible for the low $\mathrm{pH}$, but also to the production of secondary metabolites responsible for the organoleptic characteristics of the final product. In order to investigate the availability of sugars for the accomplishment of the microbial fermentation as well as the evaluation of their consumption, HPLC analysis of glucose, mannitol, sucrose and fructose was performed. The choice of these sugars was based on the fact that, according to the literature, they are the main sugars present in olives, although their concentration depends on the variety, ripeness and cultivation conditions (Kailis and Harris, 2007).

Figure 4 presents the changes of glucose and mannitol concentration in the brine during fermentations B1, B2 and B3. The concentration of glucose gradually 

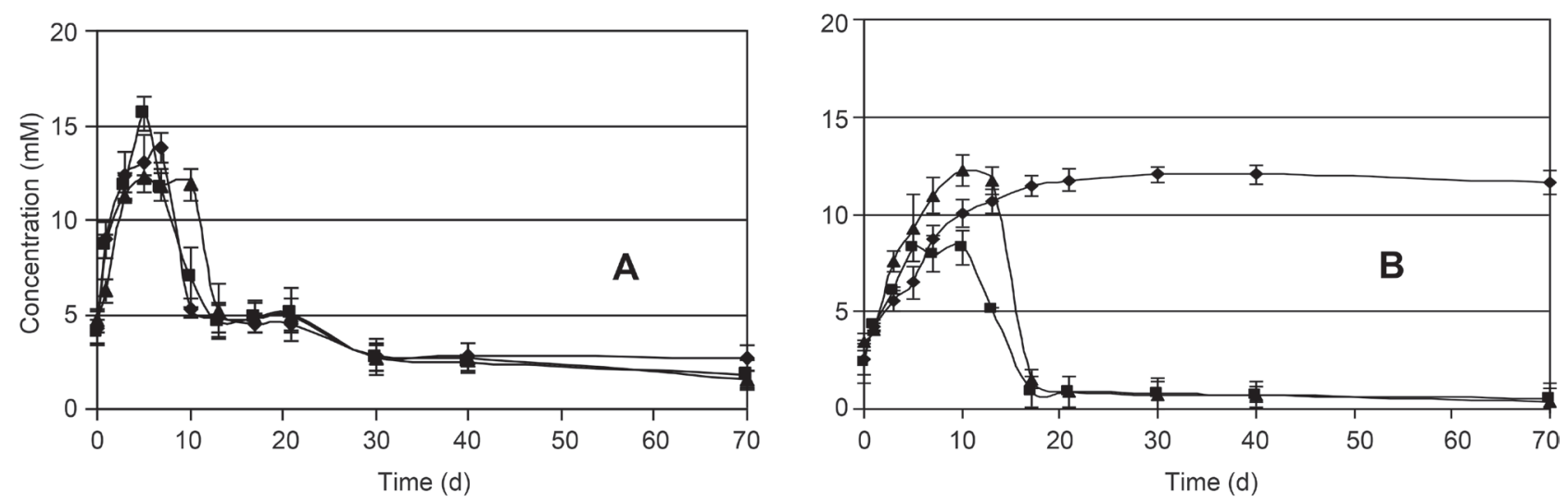

Fig. 4. Changes in the concentration (mM) of glucose (A) and mannitol (B) in brines, during the 70 days of $\mathrm{cv}$. Kalamon olives fermentation.

$(\boldsymbol{\nabla})$ : spontaneous fermentation B1, ( $\mathbf{\square})$ : fermentation B2, inoculated with L. pentosus DSM 16366, and ( $\mathbf{\Delta}$ ): fermentation B3, inoculated with L. mesenteroides subsp. mesenteroides Lm139. Data reported are means from three repetitions \pm standard deviations.

increased in all brines during the first 5-7 days, due to its diffusion from the olives to the brine, and then gradually decreased probably due to its consumption by the microorganisms. In fermentations B2 and B3 this decrease was observed earlier (at day 7) compared to fermentation B1 (at day 10). A clear difference between inoculated and non-inoculated samples was observed in the mannitol consumption which took place only in fermentations B2 and B3. Fructose was not detected at all and sucrose was detected only in traces in all three samples on day 30 and remained at the same level till the end of the fermentation period (data not shown). These two sugars were not obviously extracted from the olives, as they were not detected in the water where the olives remained for $24 \mathrm{~h}$ before being placed in the brine nor in the brines where the olives remained for $48 \mathrm{~h}$ before the addition of the starters (data not shown).

The comparison of the results obtained by monitoring of the two controlled olive fermentations B2 and $\mathrm{B} 3$ with the respective one corresponding to the spontaneous olive fermentation B1 revealed that in both cases of starters, a lactic acid fermentation was achieved by LAB. This can be supported by the growth of this microbial group solely in fermentations B2 and B3 as well as by the high lactic acid production in both inoculated samples, which led to a faster $\mathrm{pH}$ drop. The final $\mathrm{pH}$ value reached by each starter culture used (around 4.0) indicates a successful lactic fermentation that can be considered as an extra technological hurdle (Garrido-Fernández et al., 1997; Sánchez-Gómez et al., 2006; Hurtado et al., 2008).

The production of lactic acid in fermentations B2 and $\mathrm{B} 3$ as well as the concomitant drop of the $\mathrm{pH}$ value, led to the inhibition of enterobacteria in a shorter period of time compared to the spontaneous fermentation B1, as indicated by the microbiological analysis. It is already known that Gram-negative bacteria, mainly enterobacteria, dominate $\left(10^{7}-10^{8} \mathrm{cfu} \mathrm{ml}^{-1}\right)$ during the first days of olive fermentation process, till $\mathrm{LAB}$ or yeasts or both start to emerge. Then the Gram-negative bacteria disappear because of the increase of acidity due to the acid produced by the fermentation process (Garrido-Fernández et al., 1997; Sánchez-Gómez et al., 2006; Hurtado et al., 2008). Apart from the role of enterobacteria as spoilage microorganisms in table olives, their absence is also considered as an indicator of olives' safety and suitability for consumption.

On the contrary, the $\mathrm{pH}$ and the acidity values achieved as well as the absence of lactic acid in the brine of the spontaneous fermentation B1, indicate the absence of lactic fermentation and the domination of yeasts, which was confirmed by the microbiological analysis. This observation is in accordance with previous studies referring that $\mathrm{LAB}$ and yeasts compete for the fermentation of natural olives and that yeasts can be very often exclusively responsible for the fermentation (Balatsouras, 1990; Garrido-Fernández et al., 1997; Sánchez-Gómez et al., 2000; Brenes, 2004; Aponte et al., 2010). Tassou etal. (2002) reported that only yeasts were able to grow in naturally black olives (cv. Conservolea) when the $\mathrm{NaCl}$ concentration was $8 \%$.

The lactic acid fermentation accomplished in inoculated samples B2 and B3 obviously led to a faster consumption of sugars effused from the olive fruits into the brines and thus, the risk of undesirable secondary fermentations during the storage of the olives was minimized. On the contrary, the delay in the sugar consumption observed in fermentation $\mathrm{B} 1$ could be attributed to the limited fermentation activity due to the absence of LAB.

Fermentation of olives with each of the starters in brines of lower salt concentration compared to those traditionally used by the producers is important because it allows the production of Kalamata table olives with 
low salt content. Furthermore, from an environmental point of view, there is an interest in reducing the $\mathrm{NaCl}$ content of brines which are extremely difficult to treat as waste (Hurtado et al., 2012).

Concluding, the use of the LAB L.pentosus DSM 16366 and L. mesenteroides subsp. mesenteroides Lm139 as starter cultures in Kalamon olives fermentation in low salinity brine could lead to the production of olives of a constant quality, which will not depend on the indigenous microbiota that is influenced by the year and cultivation region. Such a practice could lead to a more controllable fermentation resulting in a product of high and constant quality being safer for the consumers and having a longer lifetime. As far as we know this is the first study considering the use of LAB as starter cultures during Kalamon olives fermentation, and more particularly the use of autochthonous LAB strains isolated from a spontaneous fermentation of the respective olive cultivar.

\section{Acknowledgements}

This study was funded by the "John S. Latsis Public Benefit Foundation" in the frame of "Scientific Projects 2009" program.

\section{Literature}

Aponte M., V. Ventorino, G. Blaiotta, G. Volpe, V. Farina, G. Avellone, C.M. Lanza and G. Moschetti. 2010. Study of green Sicilian table olive fermentations through microbiological, chemical and sensory analyses. Food Microbiol. 27: 162-170.

Arroyo-López F.N., A. Querol, J. Bautista-Gallego and A. Garrido-Fernández. 2008. Role of yeasts in table olive production. Int. J. Food Microbiol. 128: 189-196.

Balatsouras G.D. 1967. Processing the naturally ripe (black) olives, pp. 491-550. In: Proceedings of the International Olive Oil Seminar. Perugia-Spoleto. Italy.

Balatsouras G.D. 1990. Edible olive cultivars, chemical composition of fruit, harvesting, transportation, processing, sorting and packaging, styles of black olives, deterioration, quality standards, chemi$\mathrm{cal}$ analysis, nutritional and biological value of the end product. Proceedings of the International Seminar" Olio d'oliva e olive da tavola: tecnologia e qualità"(Istituto Sperimentale per la Elaiotecnica), pp. 291-330. Pescara. Italy.

Brenes M. 2004. Olive fermentation and processing: scientific and technological challenges. J. Food Sci. 69: 33-34.

Delgado A., D. Brito, C. Peres, F. Noé-Arroyo and A. GarridoFernández. 2005. Bacteriocin production by Lactobacillus pentosus
B96 can be expressed as a function of temperature and $\mathrm{NaCl}$ concentration. Food Microbiol. 22: 521-528.

Garrido-Fernández A., M.J. Fernández and M.R. Adams. 1997. Naturally black olives type, pp. 289-367. In: Garrido-Fernández A., M.J. Fernández and M.R. Adams (eds). Table olives: Production and processing. Chapman and Hall, London, UK.

Hurtado A., C. Reguant, B. Esteve-Zarzoso, A. Bordons and N. Rozès. 2008. Microbial population dynamics during the processing of Arbequina table olives. Food Res. Int. 41: 738-744.

Hurtado A., C. Reguant, A. Bordons and N. Rozès. 2012. Lactic acid bacteria from fermented table olives. Food Microbiol. 31: 1-8. International Olive Council (IOC). 2012a. World Table Olive Figures. http://www.internationaloliveoil.org/estaticos/view/132-worldtable-olive-figures, 2015.01.02.

International Olive Council (IOC). 2012b. The world market in figures. In: International Olive Council (ed). Olivae 117-2012: 28-34. Kailis S. and D. Harris. 2007. The olive tree Olea europaea, pp. 17-66. In: Kailis S. and D. Harris (eds). Producing Table olives. Landlinks Press, Collingwood, USA.

Marsilio V., L. Seghetti, E. Iannucci, F. Russi, B. Lanza and M. Felicioni. 2005. Use of a lactic acid bacteria starter culture during green olive (Olea europaea L cv Ascolana tenera) processing. J. Sci. Food Agr. 85: 1084-1090.

Montano A., A.H. Sanchez and A. De Castro. 1993. Controlled fermentation of Spanish-type green olives. J. Food Sci. 58: 842-844. Panagou E.Z., U. Schillinger, C.M.A.P. Franz and G.J. Nychas. 2008. Microbiological and biochemical profile of cv. Conservolea naturally black olives during controlled fermentation with selected strains of lactic acid bacteria. Food Microbiol. 25: 328-358.

Peres C., L. Catulo, D. Britoa and C. Pintadoa. 2008. Lactobacillus pentosus DSM 16366 starter added to brine as freeze-dried and as culture in the nutritive media for Spanish style green olive production. Grasas Aceites 59: 234-238.

Sánchez-Gómez A.H., A. de Castro, L. Rejano and A. Montaño. 2000. Comparative study on chemical changes in olive juice and brine during green olive fermentation. J. Agr. Food Chem. 48: 5975-5980. Sánchez-Gómez A.H., P. García and L. Rejano. 2006. Trends in table olives production, elaboration of table olives. Grasas Aceites. 57: 86-94.

Singleton V.L. and J.A. Rossi. 1965. Colorimetry of total phenolics with phosphomolybdic-phosphotungstic acid reagents. Am. J. Enol. Viticult. 16: 144-158.

Tassou C.C., E.Z. Panagou and K.Z. Katsaboxakis. 2002. Microbiological and physicochemical changes of naturally black olives fermented at different temperatures and $\mathrm{NaCl}$ levels in the brines. Food Microbiol. 19: 605-615.

Tofalo R., M. Schirone, G. Perpetuini, G. Angelozzi, G. Suzzi and A. Corsetti. 2012. Microbiological and chemical profiles of naturally fermented table olives and brines from different Italian cultivars. A. Van Leeuw. 102: 121-131.

Vlachov G. 1976. Gli acidi organici delle olive: il rapporto malico/ citrico quelle indice de maturazione. Ann. Inst. Sper. Elaiotecn. 2: 93-112. 
